\title{
Local Atomic Configuration in Pristine and A-Site Doped Silver Niobate Perovskite Antiferroelectrics
}

\author{
Jing Gao, ${ }^{1}$ Wei Li $\mathbb{D}^{1},{ }^{1}$ Jue Liu, ${ }^{2}$ Qian $\mathrm{Li} \mathbb{D}^{1},{ }^{1}$ and Jing-Feng $\mathrm{Li} \mathbb{D}^{1}$ \\ ${ }^{1}$ State Key Laboratory of New Ceramics and Fine Processing, School of Materials Science and Engineering, Tsinghua University, \\ Beijing 100084, China \\ ${ }^{2}$ Neutron Scattering Division, Oak Ridge National Laboratory, Oak Ridge, Tennessee 37831, USA
}

Correspondence should be addressed to Qian Li; qianli_mse@tsinghua.edu.cn and Jing-Feng Li; jingfeng@mail.tsinghua.edu.cn

Received 11 October 2021; Accepted 28 January 2022; Published 25 February 2022

Copyright (C) 2022 Jing Gao et al. Exclusive Licensee Science and Technology Review Publishing House. Distributed under a Creative Commons Attribution License (CC BY 4.0).

\begin{abstract}
Antiferroelectrics have attracted increasing research interests in recent years due to both their great potential in energy storage applications and intriguing structural characteristics. However, the links between the electrical properties and structural characteristics of distorted perovskite antiferroelectrics are yet to be fully deciphered. Here, we adopt local-structure methods to elucidate the nanoscale atomic structure of $\mathrm{AgNbO}_{3}$-based antiferroelectrics and their structural evolution upon La doping. The local structural features including interatomic distance distributions and atomic displacements have been analyzed using neutron small-box pair distribution function (PDF) refinement in conjunction with large-box Reverse Monte Carlo modelling. Our results highlight the correlation of cation displacements in $\mathrm{AgNbO}_{3}$ and its disruption by the incorporation of $\mathrm{La}$, apparently in corroboration with the observed anomalous dielectric properties. Spatial ordering of cation vacancies is observed in La-doped $\mathrm{AgNbO}_{3}$ samples, which coordinates with oxygen octahedral tilting to relieve lattice strain. These results provide renewed insights into the atomic structure and antiferroelectric phase instabilities of $\mathrm{AgNbO}_{3}$ and relevant perovskite materials, further lending versatile opportunities for enhancing their functionalities.
\end{abstract}

\section{Introduction}

The research and development of high efficiency energy storage materials is becoming a thriving research field. Compared to electrochemical energy storage systems such as batteries, dielectric capacitors are more favorable for applications which require high power densities, i.e., high charge/discharge speed $[1,2]$. Antiferroelectrics with antiparallel dipoles exhibit a unique reversible antiferroelectricferroelectric phase transition, upon which electrical energy is rapidly stored and released [3]. $\mathrm{AgNbO}_{3}$ and its derivatives are emergent lead-free antiferroelectrics whose great potential in energy storage applications makes them promising candidates to substitute conventional lead-containing antiferroelectrics. The intriguing structural characteristics and high energy storage properties of $\mathrm{AgNbO}_{3}$-based antiferroelectrics have obtained growing research interest from both fundamental and practical aspects [4-7]. Undoubtedly, further clarifying the correlations between structure and physical property is not only of fundamental importance but reveals also essential information for material design.

Regardless of the high energy storage property achieved thus far, little is known about the role of their structural disorder and the link to physical properties. One significant feature of the structural distortion of $\mathrm{AgNbO}_{3}$ is oxygen octahedral tilting which occurs due to the relatively small size of $\mathrm{Ag}^{+}$ion. At room temperature, the tilting follows a scheme of $\left(a^{-} b^{-} c^{-}\right) /\left(a^{-} b^{-} c^{+}\right)$in the Glazer notation [8], giving rise to a multiplied lattice of $\sqrt{2} a_{c} \times \sqrt{2} a_{c} \times 4 a_{c}$ with respect to the cubic unit cell. Another characteristic of most interest is cation displacement, which forms antiparallel dipoles along the $\pm[110]_{c}$ direction [9]. In particular, previous studies revealed that the $\mathrm{B}$-site $\mathrm{Nb}$ cation manifests additional local displacements correlated along the $\langle 001\rangle_{\text {c }}$ direction, meaning that the resultant local displacement is along the $\langle 11 \pm 1\rangle_{c}$ direction. The octahedral chains with opposite $\langle 001\rangle_{c}$ displacement are mixed spatially, and therefore, the cation displacement, on average, is along the 
$\langle 110\rangle_{\mathrm{c}}$ direction $[10,11]$. Such displacement correlations produce sheets of diffuse scattering in electron diffraction patterns [12]. Despite the conclusions achieved thus far, the exact cation displacement, especially the size and distribution of the additional [001 $]_{c}$ displacement, is still elusive, and their effects on the dielectric responses remain poorly understood.

Three polymorphs, $\mathrm{M}_{1}, \mathrm{M}_{2}$, and $\mathrm{M}_{3}$ phases, with the orthorhombic $\mathrm{Pbcm}$ space group are generally assigned to the average structure of $\mathrm{AgNbO}_{3}$ within the temperature range below $626 \mathrm{~K}$. This structure is centrosymmetric with antiferroelectric ordering [9], which however, contradicts the weak ferroelectricity experimentally observed in the $\mathrm{M}_{1}$-phased $\mathrm{AgNbO}_{3}[13,14]$. To solve this contradiction, Yashima et al. proposed the noncentrosymmetric Pmc2, model by which the antiparallel dipoles do not cancel each other completely, providing a compelling explanation for the weak ferroelectricity (ferrielectricity) [15]. Nevertheless, the subtle disparity between these two configurations renders the detection of the detailed structure of $\mathrm{AgNbO}_{3}$ rather arduous.

Chemical modification has proven an effective method to alter the phase stability of $\mathrm{AgNbO}_{3}$-based antiferroelectrics. Among the doping strategies on either A-site or B-site, La-doped $\mathrm{AgNbO}_{3}$ is of particular interest because it demonstrates substantially enhanced antiferroelectric phase stability and energy storage density compared with pure $\mathrm{AgNbO}_{3}$ [16]. Previously, it has been qualitatively observed that the lattice distortion of $\mathrm{AgNbO}_{3}$ is suppressed upon La doping, as confirmed by the decreased distortion index of cation-oxygen bond length [16]. These observations, however, are summarized from the long-range crystallographic characteristic, whereas it remains unclear how the lattice structure is altered at the local scale.

Currently, a wide range of experimental results have demonstrated that functionalities of dielectric materials depend sensitively upon their fine structural characteristics $[17,18]$. These characteristics, which are generally overlooked by conventional diffraction methods, usually exhibit some degree of deviation from the average periodicity [19, 20]. The aim of this work is to provide a comprehensive insight into the nanoscale atomic configuration of $\mathrm{AgNbO}_{3}$ with combined pair distribution functions (PDF) and the Reverse Monte Carlo (RMC) simulation method. Our results corroborate that the noncentrosymmetric $P m c 2_{1}$ space group provides a better description of the room-temperature structure of pure $\mathrm{AgNbO}_{3}$. Large atomic model is reasonably constructed using an RMC method, where the impact of $\mathrm{La}$ doping on local cation displacements is clarified. The spatial distribution of the $\langle 001\rangle_{c}$ displacement of cations yields nanoscale correlated regions, which is disrupted by La doping. The disordered nanoscale correlations are further associated with the broadening of the temperature-dependent dielectric constant in $\mathrm{La}$-doped $\mathrm{AgNbO}_{3}$. Our current work has depicted a holistic picture of the structure of $\mathrm{AgNbO}_{3}$ over multiple length scales and unravelled the generic mechanism responsible for the variation in the dielectric property, thus contributing to the design and characterization of relevant dielectric materials.

\section{Results and Discussion}

2.1. Local Structural Characteristics from PDF Refinements. Pair distribution function (PDF) proves a powerful tool for local structure characterizations of a wide variety of perovskite-structured materials [21-24]. Defined in real space, PDF contains information of both Bragg diffraction and diffuse scattering, the latter of which is usually overlooked by conventional Rietveld refinements [25]. Figures 1(a) and 1(b) present the results of neutron PDF data for $\mathrm{AN}$, where the calculated pattern is overlapped on the experimental data and a refinement is conducted on two $r$-regions of $1.5-15 \AA$ and $15-40 \AA$. Note that $15 \AA$ is a distance close to the dimension of one unit cell of $\mathrm{AgNbO}_{3}$, and thus, this dividing line is set to distinguish the local and intermediate range. The noncentrosymmetric $\mathrm{Pmc}_{1}$ and centrosymmetric $\mathrm{Pbcm}$ space groups are used as starting models for comparison. Overall, the experimental data are better fitted with the $P m c 2$, space group than $\mathrm{Pbcm}$ within both ranges. Over the distance of $1.5-15 \AA$, the refinement using $P m c 2_{1}$ improves the weighted residual $R_{w}$ from 0.1286 to 0.0679 . Similar difference, albeit less significant, is found for the $15-40 \AA$ range. The fitting to the $P m c 2_{1}$ and $P b c m$ space groups yields a $R_{w}$ factor of 0.0679 and 0.0732 , respectively, indicating that the distinction between the two configurations fades out as the scale range increases. Figure $1(\mathrm{c})$ provides a closer inspection of the low $r$-region of $1.5-5 \AA$, where the corresponding partial PDFs for specific atom pairs are presented below. The shoulder of $\mathrm{Nb}-\mathrm{O}$ pairs indicates a bimodal distribution of $\mathrm{Nb}-\mathrm{O}$ distances, which is primarily due to the off-center displacement of $\mathrm{Nb}$ ion along the $\langle 111\rangle_{\mathrm{c}}$ direction (Figure 1(d)). Particularly, the $P m c 2_{1}$ space group gives a satisfactory fit to the first nearest $\mathrm{Nb}-\mathrm{O}$ pairs, indicating that this model is more likely to fit the structure of AN with the $\langle 001\rangle_{\mathrm{c}}$ displacement at the local scale. Such compatibility with the local structure originates from the noncentrosymmetric nature of $P m c 2_{1}$ space group which removes symmetry constraints for the additional component of displacement.

However, the PDF pattern of AN at $500 \mathrm{~K}$ shows a different trend with respect to those at $300 \mathrm{~K}$. At this temperature, the $\mathrm{Pbcm}$ model provides a slightly better description than the $P m c 2_{1}$ model with $R_{w} \sim 0.11-0.13$ versus $R_{w} \sim 0.14-0.15$ (Figure S1). Refinements are also conducted for ANL4 where the data obtained at both $300 \mathrm{~K}$ and $500 \mathrm{~K}$ are considered (Figure S2). The $R_{w}$ factor of the fitting with $\mathrm{Pmc2}_{1}$ structure deteriorates with increasing temperature, whereas the fitting quality of $\mathrm{Pbcm}$ model remains little changed. Thus, both fitted $r$-regions at $500 \mathrm{~K}$ are better described by $\mathrm{Pbcm}$ structure. In particular, $\mathrm{Pbcm}$ space group describes the high $r$-region structure of both $\mathrm{AN}$ and ANL4 better than the low $r$-region, highlighting the reliability of $\mathrm{Pbcm}$ model to fit the average structure of $\mathrm{AgNbO}_{3}$-based antiferroelectrics. The overall inspection of the $R_{w}$ values indicates that the local distortion of $\mathrm{AN}$ is more suitable to be described by $P m c 2_{1}$ space group while with increasing temperature and La content, the structure evolves towards $\mathrm{Pbcm}$ structure. 


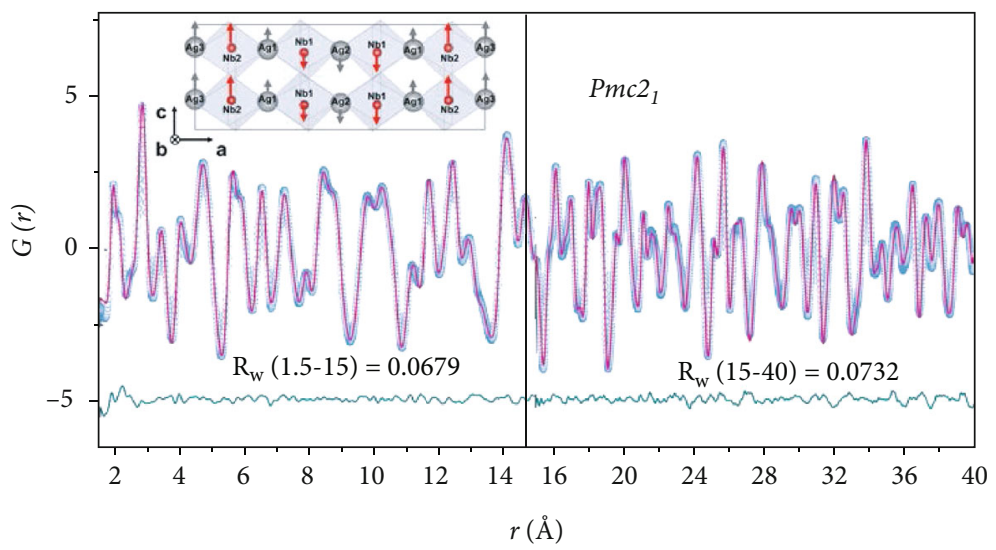

Experimental

- Calculated

— Difference

(a)

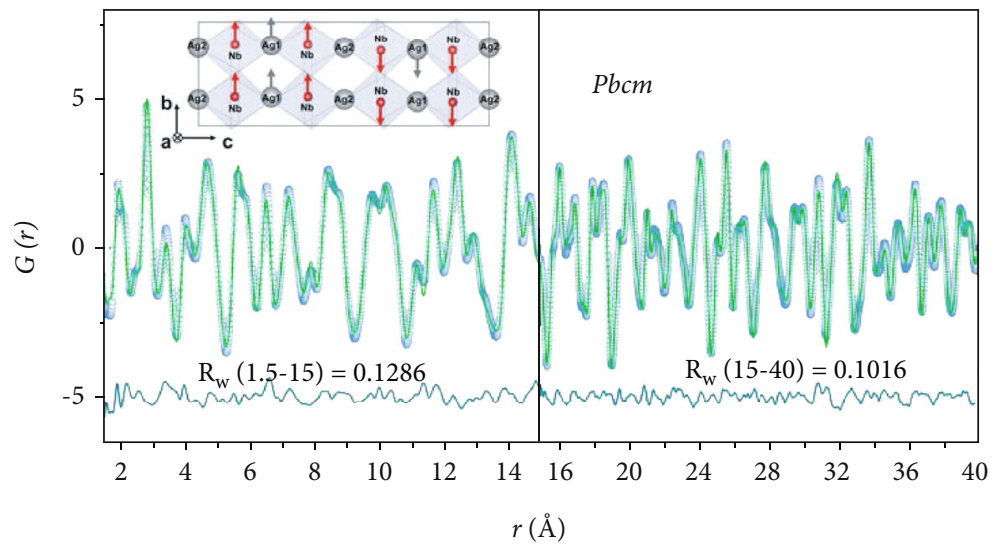

Experimental

Calculated

— Difference

(b)

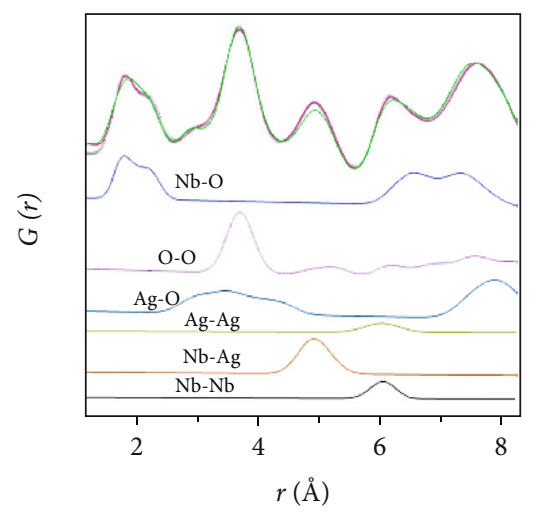

- Experimental

- $P m c 2_{1}$

- $\mathrm{Pbcm}$

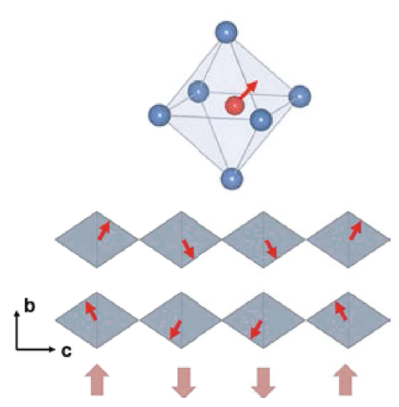

Figure 1: Room temperature PDFs of $\mathrm{AgNbO}_{3}$. (a, b) Experimental pattern fitted with Pmc21 and $\mathrm{Pbcm}$ space groups for the $r=1.5$-15 $\AA$ and the 1-40 A ranges. (c) Experimental $G(r)$ with partial PDFs of atomic pairs shown below. (d) Schematics of the local Nb displacements along the $\langle 111\rangle_{\mathrm{c}}$ direction and their correlations which give rise to the average $\langle 110\rangle_{\mathrm{c}}$ antipolar ordering. 


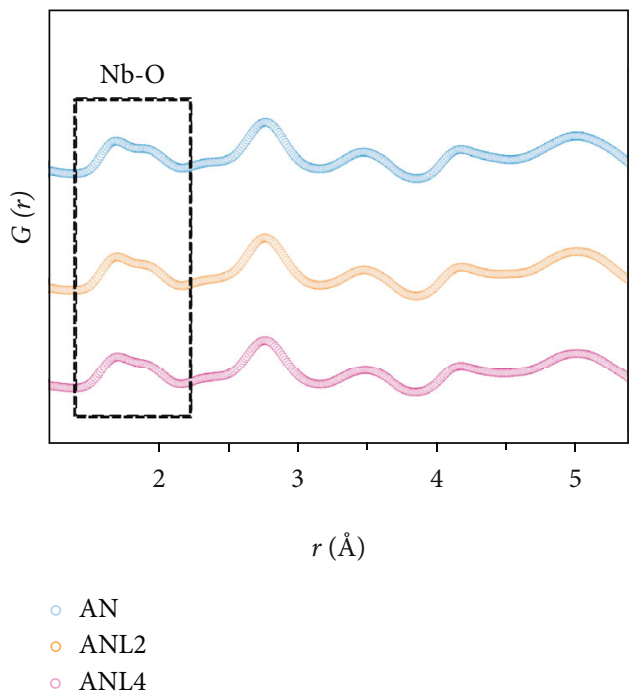

(a)

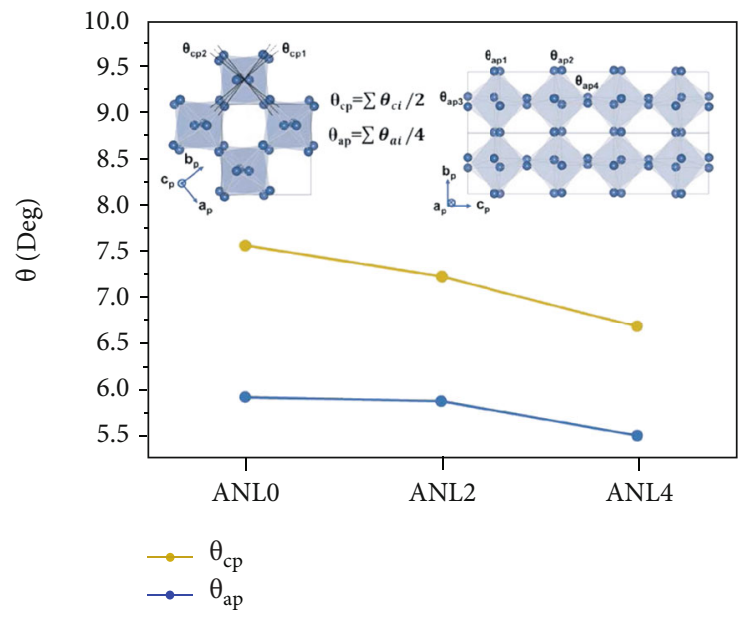

(c)

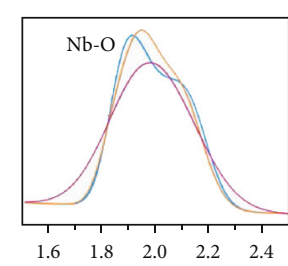

$r(\AA)$
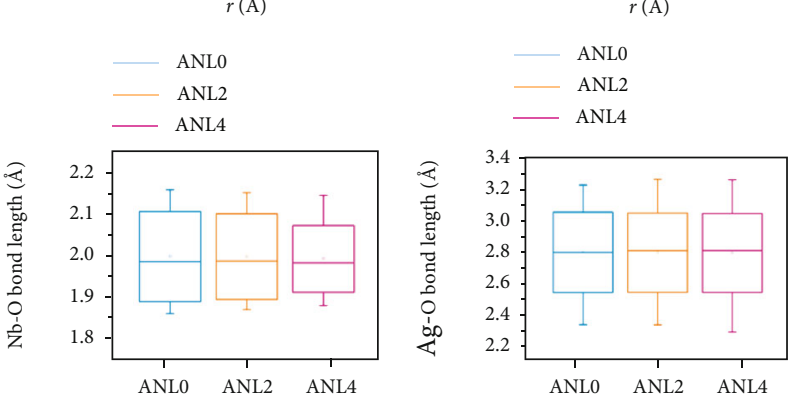

(b)

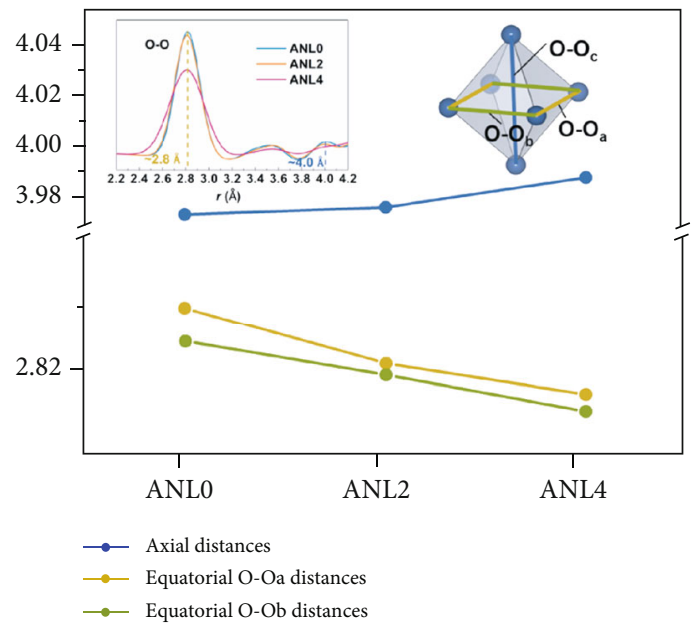

(d)

FIgURE 2: Local structural characteristics of AN, ANL2, and ANL4. (a) Experimental G( $r$ ) of AN, ANL2, and ANL4 with Nb-O pairs highlighted. (b) Partial Nb-O (upper left) and Ag-O (upper right) PDFs as obtained from PDF refinements. Box plots of Nb-O (bottom left) and Ag-O (bottom right) bond length distribution. The edges of the boxes indicate the standard deviations. (c) Oxygen octahedral tilting angles as a function of composition. The insets are schematic illustrations of $\mathrm{AgNbO}_{3}$ lattice viewed along $c_{p}$ and $a_{p}$ axes with the tilting (rotation) angles highlighted. (d) Equatorial $\mathrm{O}-\mathrm{O}_{\mathrm{a}}$ and $\mathrm{O}-\mathrm{O}_{\mathrm{b}}$ distances and axial $\mathrm{O}-\mathrm{O}_{\mathrm{c}}$ distances as a function of composition. The inset shows the partial O-O PDFs and the $\left[\mathrm{NbO}_{6}\right]$ octahedron with specific $\mathrm{O}-\mathrm{O}$ distances marked.

To shed light on the effect of La dopant on the local and intermediate structure of $\mathrm{AgNbO}_{3}$, the low $r$-region PDFs for AN, ANL2, and ANL4 are compared, as shown in Figure 2(a). Small but yet systematic differences are observed for the Nb-O pairs. The shoulder peak is gradually smeared from AN to ANL4, suggesting a more uniform distribution of Nb-O bond lengths. Partial Nb-O PDFs for different compositions are shown in Figure 2(b), and the $\mathrm{Nb}-\mathrm{O}$ bond length distribution summarized from the calculated model is plotted below. Appreciable narrower spread of the $\mathrm{Nb}-\mathrm{O}$ distances with increasing La content is observed, implying the suppressed off-center displacement of $\mathrm{Nb}^{5+}$. The Ag-O bond lengths, by contrast, show a more distorted coordination with the length values ranging between $2.3 \AA$ and $3.2 \AA$. The highly distorted $\mathrm{Ag}^{+}$displacement is primarily associated with its interstitial position, suggesting that $\mathrm{Ag}^{+}$ cations are more flexible than $\mathrm{Nb}^{5+}$ to accommodate the ionic size mismatch.

Apart from cation displacements, oxygen octahedral tilting is another significant structural characteristic. The calculated oxygen octahedral tilting angles of $\mathrm{AN}$ and their composition dependence are shown in Figure 2(c), along with schematics for the designation of tilting angles with respect to the pseudo perovskite $c_{p}$ and $a_{p}$ axis. These two types of tilting angles decrease with increasing La content, suggesting a suppressed octahedral tilting scheme. The decreased magnitude of tilting angles indicates a structural relaxation which occurs primarily to mitigate the undercoordination of oxygen. Besides, given that the change of octahedral tilting will inevitably alter the interatomic forces 
between the neighbouring cations, one can expect a notable correlation between the decreased octahedral tilting angle and suppressed cation displacement.

Due to the high sensitivity of neutron scattering to oxygen, the $\mathrm{O}^{2-}$ positions can be reliably characterized, facilitating analyses of the evolution of individual oxygen octahedron upon doping. Two types of $\mathrm{O}-\mathrm{O}$ distances, that is, the distances in the (001) plane and the distances along the $<001>$ direction are focused. The equatorial and axial O-O distances are around $2.8 \AA$ and $4 \AA$, respectively, as highlighted by the partial O-O PDFs in Figure 2(d) Inset. The equatorial O-O distances are divided into two groups labelled $\mathrm{O}-\mathrm{O}_{\mathrm{a}}$ and $\mathrm{O}-\mathrm{O}_{\mathrm{b}}$. Interestingly, the equatorial O-O distances show a progressive contraction while the axial $\mathrm{O}-\mathrm{O}$ distances follow an opposite trend, exhibiting an anisotropic variation upon La doping. The extended axial O-O distances, combined with the suppressed tilting along $c_{p}$ axis, account for the experimental observation that the lattice parameter $c_{p}$ shows progressive increase with increasing $\mathrm{La}$ content (Figure S3). Interestingly, the anisotropic change of the shape of the oxygen octahedron is consistently observed upon the change of temperature [10]. This suggests that, to a certain extent, La doping and elevated temperature modify the shape of individual oxygen octahedron in parallel ways.

2.2. Cation Displacement. To comprehensively understand the spatial variations of the atomic structure, RMC protocols are implemented to model the large length-scale atomic configurations. First, we compared the fitting result with the initial configuration of $\mathrm{Pbcm}$ and $\mathrm{Pmc2}_{1}$ models. Though both models yield satisfactory results, the computation with the $\mathrm{Pbcm}$ model has reached a lower loss in both $G(r)$ and $S(Q)$ fitting, and the results are more consistent with the experimental data, compared with those using the $P m c 2_{1}$ model (see Figure S4). We assume that the higher symmetry of $\mathrm{Pbcm}$ space group makes it easier for the algorithm to find the initial moves during the early stage of fitting. Additionally, the subtle difference in ionic displacements between the two models makes no discernible influence (as has been confirmed) after random atom moves occur in RMC fitting process. Thus, all the RMC simulation results reported here are from the $\mathrm{Pbcm}$ initial model. The Bragg profiles and neutron total scattering data in both real and reciprocal spaces were fitted simultaneously, and the refined configuration provided a satisfactory fit to all datasets (Figure S5). AN and ANL4 are selected hereafter for comparison to highlight the effect of La dopant on the structural evolution.

For a clear demonstration, the spatial displacement of cations is decomposed into an in-plane component (displacement within the $a b$ plane) plus an out-of-plane one (displacement along the $c$ axis). The map of the in-plane displacement of $\mathrm{Nb}^{5+}$ for $\mathrm{AN}$ is shown in Figure 3(a), where several regions of correlated orientation can be seen. Figure 3(b) compares the profile of the displacement distribution along the horizontal direction for AN and ANL4, where a marginally decreased off-center displacement is observed for ANL4. Figures 3(c) and 3(d) depict the distri- bution of $\mathrm{Nb}^{5+}$ displacement for AN, which exhibits an obvious two-site preferred distribution along the $\langle 110\rangle_{c}$ direction, mirroring their antiparallel displacement. The magnitude of the antipolar displacement is around $10 \mathrm{pm}$, comparable to the experimental observations by scanning transmission electron microscopy [26]. For ANL4, the antipolar pattern is mostly retained but with a narrowed spread (Figure 3(d)). This phenomenon is generally consistent with the suppressed distortion of the $\mathrm{Nb}-\mathrm{O}$ bond, as suggested above by the small-box PDF refinement.

The distribution of Ag appears to be more complicated because there are two distinct sites for Ag, i.e., Ag1 and Ag2. Figures S6(a) and S6(b) present the maps of the Ag1 and $\mathrm{Ag} 2$ displacement for AN. The distribution of Ag1 follows a bimodal scenario while the distribution of $\mathrm{Ag} 2$ appears to be unimodal, which is consistent with the $\mathrm{Pbcm}$ symmetry. As for ANL4, the displacement of Ag is arranged in a similar way to that of AN while its distribution becomes narrower (Figures S6(c) and S6(d)). This contrast is further ascertained by the sharper peaks for ANL4 in corresponding profiles (Figure S6(e) and S6(f)). The above observations agree with the known trends that La doping tends to suppress the cation displacement in $\mathrm{AgNbO}_{3}$, primarily due to its less covalent bonding with oxygen [5].

Next, we extract the $\langle 001\rangle_{\mathrm{c}}$ displacement of $\mathrm{Nb}^{5+}$ for analysis, and the configuration is visualized in Figure 4 . Interestingly, the distribution of the $\langle 001\rangle_{c}$ displacement of $\mathrm{Nb}$ cations in $\mathrm{AN}$ is not completely random but rather displays certain degree of aggregation, featuring correlated regions several nanometers in size, inside which the cation displacements are along the same direction, as highlighted by the color contrast in Figure 4(a). Within these nanoregions, the maximum displacement of $\mathrm{Nb}^{5+}$ ions is about $30 \mathrm{pm}$, and the clusters are around 6-10 nm in size. Nanoregions are observed for the $\langle 001\rangle_{c}$ displacement of $\mathrm{Ag}$ cations as well with a notable coupling with that of $\mathrm{Nb}$ cations (Figure S7). As expected, Ag cations exhibit larger displacements than $\mathrm{Nb}$ within the same regions, conforming to the larger distortion of $\mathrm{Ag}-\mathrm{O}$ as suggested by the PDF refinement.

In contrast to the correlated nanoregions in pure $\mathrm{AN}$, the distribution of the $\langle 001\rangle_{c}$ displacement of $\mathrm{Nb}$ cations appears to be more random and fragmental for ANL4 (Figure 4(b)), implying that the incorporation of La disrupts the short-range order of the cation displacements. In addition to the weaker correlation, the maximum magnitude of the displacements also decreases to $20 \mathrm{pm}$. Inspection of the configuration for Ag cations demonstrates a similar disordered pattern in ANL4 (Figure S7(b)). For more details, the supercell with a height of $12 \mathrm{~nm}$ is sliced into 32 layers along the $z$-direction (each $3.75 \AA$ thick, close to the scale of one unit cell), and the projection of the $\langle 001\rangle_{c}$ displacement of each layer is presented in Figure S8. Evidently, the coupled clusters of the $\langle 001\rangle_{\mathrm{c}}$ displacement have been broken in ANL4. With the disappearance of correlated clusters, the distribution of the displacement also becomes smaller in magnitude. These observations suggest that La doping alters the cation displacement of $\mathrm{AgNbO}_{3}$ in both magnitude and distribution. 


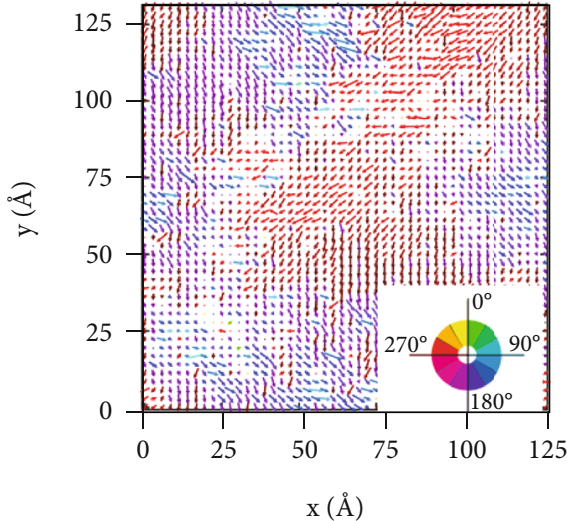

(a)

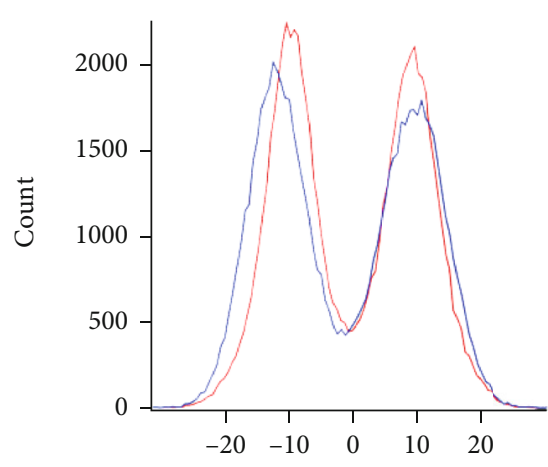

$\Delta \mathrm{x}(\mathrm{pm})$

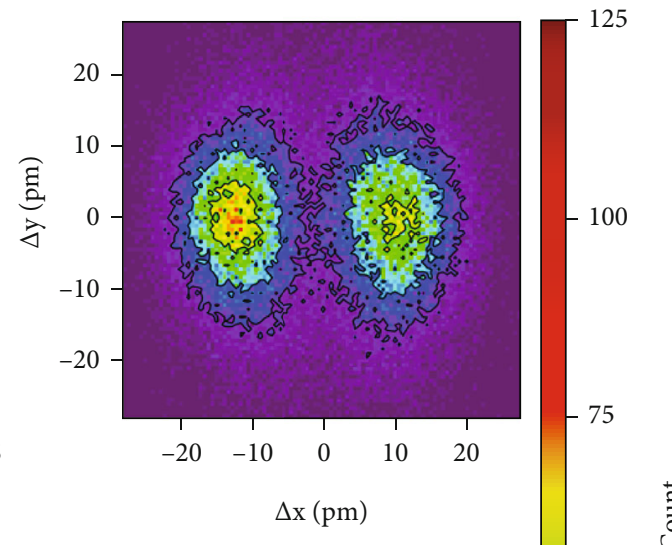

(c)

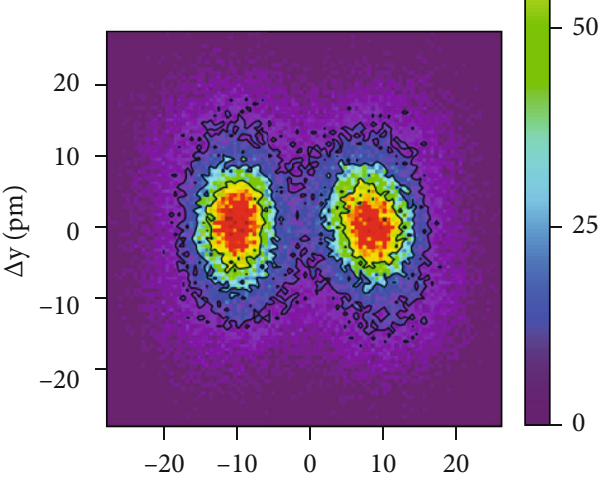

$\Delta \mathrm{x}(\mathrm{pm})$

(d)

FIGURE 3: A complete view of the displacement of $\mathrm{Nb}$ atom projected onto the (110) plane (in-plane displacement). (a) Illustration of the $\mathrm{Nb}$ displacement; the arrows point the displacement direction, and the length represents the magnitude of the displacement. (b) Comparison of profiles of $\mathrm{Nb}$ in-plane displacement for AN and ANL4. (c, d) Illustration of the projection of Nb displacements of AN and ANL4, respectively.

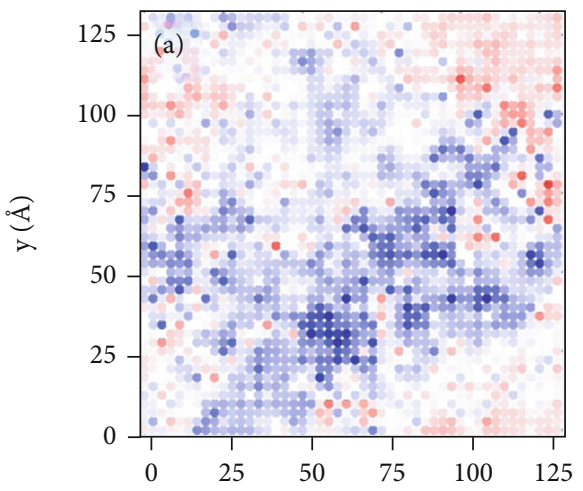

$\mathrm{x}(\AA)$

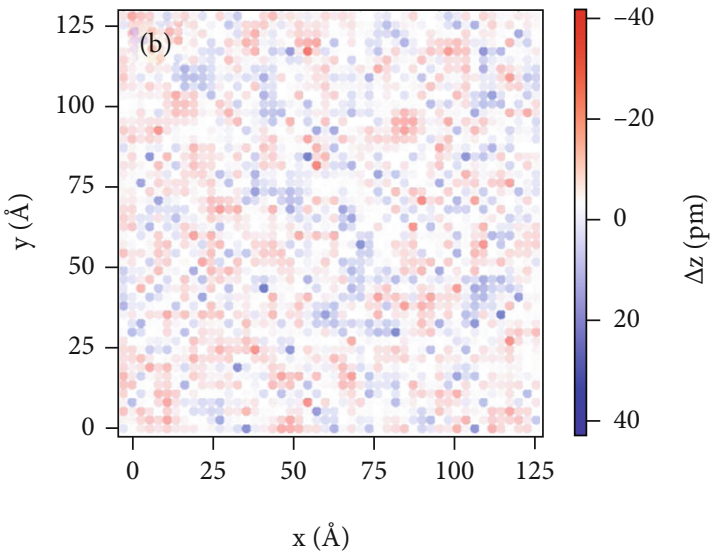

FIGURE 4: Projection of the $\mathrm{Nb}\langle 001\rangle_{\mathrm{c}}$ displacement. $\langle 001\rangle_{\mathrm{c}}$ displacement for (a) AN and (b) ANL4. The color-scale bar reflects the magnitude of the displacement. The horizontal and vertical axes correspond to the $\langle 100\rangle$ and $\langle 010\rangle$ directions, respectively. 
2.3. A-Site Vacancy Ordering. In $\mathrm{Ag}_{1-3 \mathrm{x}} \mathrm{La}_{\mathrm{x}} \mathrm{NbO}_{3}$, for each $\mathrm{La}$ that substitutes for Ag, two A-site vacancies will be induced to maintain charge neutrality. Generally, at sufficiently high concertation, vacancies are prone to aggregate in order to minimize the free energy of the system $[27,28]$. The configuration of A-site vacancies in ANL4 is explored by analyzing the supercell reconstructed by the RMC method. As shown in Figure 5(a), the A-site vacancies of ANL4 are preferentially distributed in every twice $[001]_{c}$ row to produce alternative layers with vacant and occupied A-site positions. Figure 5(b) displays the corresponding histogram for the distribution of A-site vacancies. Upon close examination, the vacancies show an obvious preference for the Ag2 position whereas the probability of its presence at the Ag1 position is low. This suggests that the nonequivalent coordination environment of A-site cations could be the origin of the distinct array of vacancies. The ordering of $\mathrm{Ag}$ vacancies is reminiscent of analogous A-site-deficient perovskites in which the A-site vacancies serve as mediators to relieve bond strain of the lattice $[29,30]$. Layered ordering of the A-site cations is commonly observed in cationdeficient perovskites such as $\mathrm{La}_{1 / 3} \mathrm{NbO}_{3}$ and $\mathrm{La}_{2 / 3} \mathrm{TiO}_{3}$ [31, 32]. These A-site-deficient compounds usually contain modulated structures with alternating (001) layers of fully and partly occupied A-sites. Second-order Jahn-Teller effect, where highly charged $\mathrm{d}^{0}$ cations on the B-site moves out of the center of their coordination polyhedral, is an important mechanism for stabilizing A-site cation ordering and relieving the bonding instability caused by ordering [33, 34]. Therefore, it is likely that for La-doped $\mathrm{AgNbO}_{3}$, the specific ordering of A-site vacancies and variation in oxygen octahedral tilting angle generates a cooperative effect to stabilize the structure. It should be noted that the analysis of vacancy ordering based on RMC describes the models of reasonable probability, while multiple competing vacancy configurations are likely to coexist due to the abundant defects in polycrystalline ceramics and thermal fluctuations on ambient conditions [35]. More deterministic information might be obtained if the energy difference is enlarged when the concentration of vacancies is high enough to populate a significantly distinct configuration.

2.4. Dielectric Properties. The disrupted nanoscale correlations upon La doping appear to induce a gradual change in the dielectric properties. Figure S9 shows the temperaturedependent dielectric constant of AN, ANL2, and ANL4 measured on heating and cooling, respectively. An anomaly that is called freezing temperature $[36,37] T_{f}$ is observed for $\mathrm{AN}$ at $\sim 175^{\circ} \mathrm{C}$, which becomes greatly smeared for ANL2 and ANL4. Generally, $T_{f}$ indicates the hopping dynamics of $\mathrm{Nb}^{5+}$ among the eight $\langle 111\rangle_{\mathrm{c}}$ positions in the lattice. Below $T_{f}$, the two sites along the $\langle 110\rangle_{\mathrm{c}}$ direction are preferentially occupied thus initiating the antiparallel order of $\mathrm{Nb}^{5+}$ displacements [38]. This mechanism implies that $T_{f}$ is closely associated with cation displacements, especially the $\langle 001\rangle_{c}$ displacement correlations. It is thus reasonable to assume that the disrupted nanoscale correlations are the primary origin of the smeared $T_{f}$ of

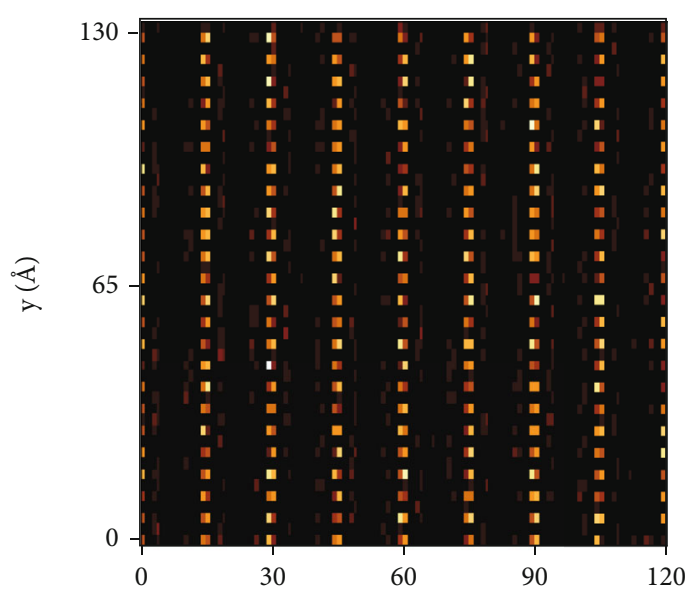

(a)

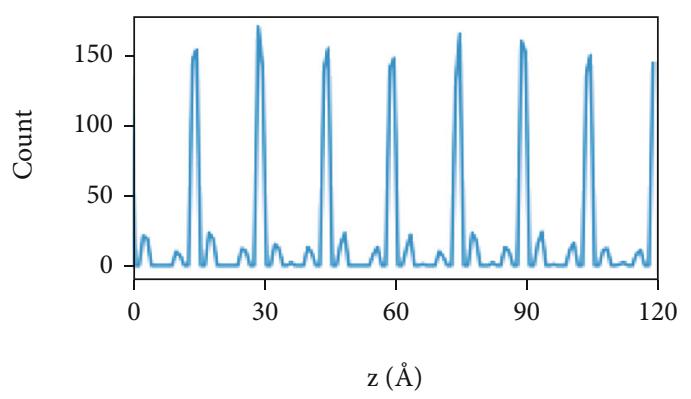

(b)

FIgURE 5: An illustration of ordered A-site vacancies in ANL4. The $y$ and $z$ axes correspond to the $\langle 010\rangle$ and $\langle 001\rangle$ directions, respectively. (a) An in-plane detailed view of vacancy positions. (b) Histogram of vacancies on different layers.

ANL2 and ANL4. Considering the fact that the eight-site model of cation displacement is commonly observed in other perovskite compounds such as $\mathrm{NaNbO}_{3}$ [30] and $\mathrm{PbMg}_{1 / 3} \mathrm{Nb}_{2 / 3} \mathrm{O}_{3}$ [17], the scenario proposed here may convincingly account for the displacive disorder in these relevant perovskites and corresponding variation in dielectric properties.

\section{Conclusion}

In summary, small-box refinement and large-box simulation have been incorporated to examine the structural characteristics of pure and $\mathrm{La}$-doped $\mathrm{AgNbO}_{3}$ at different length scales. Modelling of the PDF reveals that the noncentrosymmetric space group $P m c 2_{1}$ provides a better description of the room-temperature structure of $\mathrm{AgNbO}_{3}$. The incorporation of La tends to suppress the structural distortion with respect to bond length distributions as well as octahedral tilting. The large atomic configuration established by the RMC method demonstrates that the antipolar displacement of cations within the $(001)_{\mathrm{c}}$ plane are suppressed upon La doping. The displacements of $\mathrm{Nb}$ and $\mathrm{Ag}$ along the $\langle 001\rangle_{\mathrm{c}}$ direction are qualitatively coherent in pure $\mathrm{AgNbO}_{3}$, and the alignment of these clusters gives rise to correlated nanoregions several nanometers in size, which appears to be disrupted 
in La-doped $\mathrm{AgNbO}_{3}$. The disordered nanoscale correlations of cations suppress the anomaly of dielectric constant around the freezing temperature. A-site vacancies induced by the aliovalent dopants are preferentially displaced at the Ag2 site and tend to order into alternate (001) layers. Our work has revealed that local structural characteristics and their assembly into the long-range average configurations are essential to depict a clear picture of the atomic structure of $\mathrm{AgNbO}_{3}$. These insights provide guidelines for future exploration of the structural characteristics of perovskitebased antiferroelectrics as well as the underlying structureproperty correlation.

\section{Materials and Methods}

4.1. Synthesis and Measurement of Dielectric Properties. Ceramic samples of pure and La-doped $\mathrm{AgNbO}_{3}$ $\left(\mathrm{Ag}_{1-3 \mathrm{x}} \mathrm{La}_{\mathrm{x}} \mathrm{NbO}_{3}\right)$ were prepared by solid-state reaction according to the synthesis route described in references [16, 39]. Here, three compositions, $\mathrm{AgNbO}_{3}, \mathrm{Ag}_{0.94} \mathrm{La}_{0.02} \mathrm{NbO}_{3}$, and $\mathrm{Ag}_{0.88} \mathrm{La}_{0.04} \mathrm{NbO}_{3}$ (abbreviated as AN(ANL), ANL2, and ANL4, respectively), were specifically chosen for detailed structural characterization.

For the measurement of dielectric properties, the samples were coated with Ag electrodes, and the measurement was conducted in a furnace using an LCR meter (TH2828S; Changzhou Tonghui).

4.2. Total Scattering Measurements. Neutron total scattering data were collected at both $300 \mathrm{~K}$ and $500 \mathrm{~K}$ at Spallation Neutron Source (SNS)'S NOMAD beamline of Oak Ridge National Laboratory. The ceramic pellets of different compositions were crushed into powders, followed by annealing at $500^{\circ} \mathrm{C}$ for $3 \mathrm{~h}$ in order to remove possible residual stresses, and the powders were then sealed in quartz capillaries for measurement. The total scattering data cutoff at a maximum wavevector transfer $Q_{\max }$ of $40 \AA^{-1}$ were used for Fourier transform to the reduced pair distribution function. Smallbox modelling of the PDF data was conducted with PDFgui packages [40]. The samples employed in this work were processed from the same batch as in ref. [16], where the phase purity was confirmed by neutron diffraction. In the unit cell of ANL2 and ANL4 composition, 2 mol\% or $4 \mathrm{~mol} \% \mathrm{La}$ atoms were put at the A-site with no preference for the distinct crystallographic Ag sites.

4.3. Structure Refinements. Large-box modelling was performed by Reverse Monte Carlo (RMC) simulations with the RMCprofile software [41] using a $24 \times 24 \times 8$ supercell $\left(\sim 13.3 \times 13.4 \times 12.5 \mathrm{~nm}^{3}\right)$ containing 184320 atoms. Specifically, the model for AN contains $36864 \mathrm{Ag}, 36864 \mathrm{Nb}$, and $110592 \mathrm{O}$ atoms. For ANL4, $32558 \mathrm{Ag}, 36864 \mathrm{Nb}, 110592 \mathrm{O}$, $1475 \mathrm{La}$, and $2831 \mathrm{~V}_{\mathrm{a}}$ (vacancies) were included in the model. La atoms and vacancies were introduced by randomly changing $4 \%$ of $\mathrm{Ag}$ atoms to $\mathrm{La}$ atoms and $8 \%$ of $\mathrm{Ag}$ atoms to vacancies at the same time. Positions of La atoms, $\mathrm{Ag}$ atoms, and vacancies are allowed to swap with $20 \%$ probability. Various simulation durations were adopted according to certain parameter settings and corresponding convergence rates. About 15 million moves were generated $(\sim 1$ million accepted) per the algorithm to simultaneously fit the Bragg profiles and neutron total scattering data in both real and reciprocal spaces. For most simulation parameters, the default settings are adopted. We use atom distance (defined as the distance window in RMCprofile) to constrain atom moves. During the simulations, weights between Bragg profiles and neutron total scattering data were adjusted dynamically according to their fitting condition.

Additional RMC simulations were performed to verify the reliability of the fitting result. The fitting was separated to two stage. In the first stage, the atom moving range was set at $0.8 \AA$. The cutoff boundary was decided correspond to $G(r)$ profiles. An automated optimization of weights among the $G(r), S(Q)$, and Bragg profiles was enabled. The program was allowed to accept some moves that would make the loss rise slightly. In the second stage, the atom moves were limited to $0.2 \AA$, and the optimization process was cancelled. The evolution of acceptance rate and fitting loss was recorded to evaluate the influence of stage 1 . Similar conclusions of atom displacement and vacancy arrangement could be drawn, and the validity of the RMC model is solid. See more details in Figure S11 and S12 and discussions therein.

\section{Data Availability}

The data used to support the findings of this study are available from the corresponding authors upon request.

\section{Conflicts of Interest}

The authors declare no conflicts of interest.

\section{Authors' Contributions}

This study was conceived and designed by JFL and QL. JG and JFL synthesized samples and performed dielectric measurements. JL and QL performed neutron PDF measurements. WL, JG, and QL analyzed the PDF results. JG and WL cowrote the manuscript with input from all authors. Jing Gao and Wei Li contributed equally to this work.

\section{Acknowledgments}

The authors acknowledge useful discussions with Yun Liu, Terry Frankcombe, and Yuanpeng Zhang. This work was supported by the Basic Science Center Project of NSFC under Grant No. 51788104, NSFC under Grant No. 52073155, and Tsinghua-Foshan Innovation Special Fund (TFISF) under Grant No. 2020THFS0113. Research conducted at the NOMAD beamlines at ORNL's Spallation Neutron Source was sponsored by the Scientific User Facilities Division, Office of Basic Sciences, U.S. Department of Energy.

\section{Supplementary Materials}

Figure S1: PDFs of AN ( $500 \mathrm{~K})$. Figure S2: PDF refinements for ANL4. Figure S3: Lattice parameter $c_{p}$ as a function of La 
content. Figure S4: a comparison of the fitting quality between calculations with starting model of $\mathrm{Pbcm}$ and Pmc21 space groups. Figure S5: results of data fitting for $\mathrm{AN}$ and ANL4 at $300 \mathrm{~K}$. Figure S6: illustration of the projection of Ag displacements. Figure S7: projection of the $\langle 001\rangle_{\mathrm{c}}$ displacement of Ag. Figure S8: overall view of the $\langle 001\rangle_{\mathrm{c}} \mathrm{Nb}$ atom displacement. Figure S9: overall view of the $\langle 001\rangle_{\mathrm{c}} \mathrm{Ag}$ atom displacement. Figure S10: temperature-dependent dielectric constant of AN, ANL2, and ANL4. Figure S11: RMC fitting results for the verification test. Figure S12: analysis for the RMC verification test. (Supplementary Materials)

\section{References}

[1] Z. Yao, Z. Song, H. Hao et al., "Homogeneous/inhomogeneous-structured dielectrics and their energy-storage performances," Advanced Materials, vol. 29, no. 20, p. 1601727 , 2017.

[2] L. Yang, X. Kong, F. Li et al., "Perovskite lead-free dielectrics for energy storage applications," Progress in Materials Science, vol. 102, pp. 72-108, 2019.

[3] Z. Liu, T. Lu, F. Xue et al., "Lead-free (Ag,K)NbO3materials for high-performance explosive energy conversion," Science Advances, vol. 6, no. 21, article eaba0367, 2020.

[4] N. Luo, K. Han, M. J. Cabral et al., "Constructing phase boundary in $\mathrm{AgNbO}_{3}$ antiferroelectrics: pathway simultaneously achieving high energy density and efficiency," Nature Communications, vol. 11, no. 1, p. 4824, 2020.

[5] J. Gao, Q. Li, S. Zhang, and J.-F. Li, "Lead-free antiferroelectric $\mathrm{AgNbO}_{3}$ : phase transitions and structure engineering for dielectric energy storage applications," Journal of Applied Physics, vol. 128, no. 7, article 070903, 2020.

[6] L. Zhao, Q. Liu, J. Gao, S. Zhang, and J. F. Li, "Lead-free antiferroelectric silver niobate tantalate with high energy storage performance," Advanced Materials, vol. 29, no. 31, p. 1701824, 2017.

[7] D. Yang, J. Gao, L. Shu et al., "Lead-free antiferroelectric niobates $\mathrm{AgNbO}_{3}$ and $\mathrm{NaNbO}_{3}$ for energy storage applications," Journal of Materials Chemistry A, vol. 8, no. 45, pp. 2372423737, 2020.

[8] A. Glazer, "The classification of tilted octahedra in perovskites," Acta Crystallographica, Section B: Structural Crystallography and Crystal Chemistry, vol. 28, no. 11, pp. 33843392, 1972.

[9] P. Sciau, A. Kania, B. Dkhil, E. Suard, and A. Ratuszna, "Structural investigation of $\mathrm{AgNbO}_{3}$ phases using X-ray and neutron diffraction," Journal of Physics: Condensed Matter, vol. 16, pp. 2795-2810, 2004.

[10] I. Levin, V. Krayzman, J. C. Woicik et al., "Structural changes underlying the diffuse dielectric response in $\mathrm{AgNbO}_{3}$," Physical Review B, vol. 79, no. 10, p. 104113, 2009.

[11] I. Levin, J. C. Woicik, A. Llobet et al., "Displacive ordering transitions in perovskite-like $\mathrm{AgNb}_{1 / 2} \mathrm{Ta}_{1 / 2} \mathrm{O}_{3}$," Chemistry of Materials, vol. 22, no. 17, pp. 4987-4995, 2010.

[12] V. Krayzman and I. Levin, "Reverse Monte Carlo refinements of local displacive order in perovskites: $\mathrm{AgNbO}_{3}$ case study," Journal of Physics. Condensed Matter, vol. 22, no. 40, p. 404201, 2010.

[13] A. Kania, K. Roleder, and M. Łukaszewski, “The ferroelectric phase in $\mathrm{AgNbO}_{3}$," Ferroelectrics, vol. 52, no. 1, pp. 265-269, 1983.
[14] D. Fu, M. Endo, H. Taniguchi, T. Taniyama, and M. Itoh, " $\mathrm{AgNbO}_{3}$ : a lead-free material with large polarization and electromechanical response," Applied Physics Letters, vol. 90, no. 25, p. 252907, 2007.

[15] M. Yashima, S. Matsuyama, R. Sano, M. Itoh, K. Tsuda, and $\mathrm{D}$. Fu, "Structure of ferroelectric silver niobate $\mathrm{AgNbO}_{3}$," Chemistry of Materials, vol. 23, no. 7, pp. 16431645, 2011.

[16] J. Gao, Y. Zhang, L. Zhao et al., "Enhanced antiferroelectric phase stability in La-doped $\mathrm{AgNbO}_{3}$ : perspectives from the microstructure to energy storage properties," Journal of Materials Chemistry A, vol. 7, no. 5, pp. 2225-2232, 2019.

[17] M. Eremenko, V. Krayzman, A. Bosak et al., "Local atomic order and hierarchical polar nanoregions in a classical relaxor ferroelectric," Nature Communications, vol. 10, no. 1, p. 2728, 2019.

[18] P. K. Davies and M. A. Akbas, "Chemical order in PMNrelated relaxors: structure, stability, modification, and impact on properties," Journal of Physics and Chemistry of Solids, vol. 61, no. 2, pp. 159-166, 2000.

[19] D. A. Keen and A. L. Goodwin, "The crystallography of correlated disorder," Nature, vol. 521, no. 7552, pp. 303-309, 2015.

[20] L. Jiang, D. C. Mitchell, W. Dmowski, and T. Egami, "Local structure of $\mathrm{NaNbO}_{3}$ : a neutron scattering study," Physical Review B, vol. 88, no. 1, article 014105, 2013.

[21] I. Levin, D. S. Keeble, G. Cibin et al., "Nanoscale polar heterogeneities and branching bi-displacement directions in $\mathrm{K}_{0.5} \mathrm{Bi}_{0.5} \mathrm{TiO}_{3}$," Chemistry of Materials, vol. 31, no. 7, pp. 2450-2458, 2019.

[22] B. Jiang, T. Grande, and S. M. Selbach, "Local structure of disordered $\mathrm{Bi}_{0.5} \mathrm{~K}_{0.5} \mathrm{TiO}_{3}$ investigated by pair distribution function analysis and first-principles calculations," Chemistry of Materials, vol. 29, no. 10, pp. 4244-4252, 2017.

[23] B. Jiang and S. M. Selbach, "Local and average structure of Mnand La-substituted $\mathrm{BiFeO}_{3}$," Journal of Solid State Chemistry, vol. 250, pp. 75-82, 2017.

[24] H. Y. Playford, L. R. Owen, I. Levin, and M. G. Tucker, "New insights into complex materials using reverse Monte Carlo modeling," Annual Review of Materials Research, vol. 44, no. 1, pp. 429-449, 2014.

[25] T. Egami and S. J. Billinge, Underneath the Bragg Peaks: Structural Analysis of Complex Materials, Elsevier, 2003.

[26] G. Li, H. Liu, L. Zhao et al., "Atomic-scale structure characteristics of antiferroelectric silver niobate," Applied Physics Letters, vol. 113, no. 24, p. 242901, 2018.

[27] S. García-Martín and M. Á. Alario-Franco, "Modulated structure of $\mathrm{La}_{1 / 3-\mathrm{x}} \mathrm{Li}_{3 \mathrm{x}} \mathrm{NbO}_{3} \quad 0 \leq \mathrm{x} \leq 0.06$ perovskite-related materials," Journal of Solid State Chemistry, vol. 148, no. 1, pp. 93-99, 1999.

[28] X. Hu, S. Kobayashi, Y. H. Ikuhara et al., "Atomic scale imaging of structural variations in $\mathrm{La}_{(1-\mathrm{x}) / 3} \mathrm{Li}_{\mathrm{x}} \mathrm{NbO}_{3}(0 \leq \mathrm{x} \leq 0.13)$ solid electrolytes," Acta Materialia, vol. 123, pp. 167-176, 2017.

[29] I. Levin, V. Krayzman, H. Y. Playford et al., "The mediation of bond strain by vacancies and displacive disorder in A-sitedeficient perovskites," Acta Materialia, vol. 207, p. 116678, 2021.

[30] I. Levin, F. Yang, R. Maier et al., "Displacive order-disorder behavior and intrinsic clustering of lattice distortions in bisubstituted $\mathrm{NaNbO}_{3}, "$ Advanced Functional Materials, vol. 30, no. 30, p. 2001840, 2020. 
[31] B. J. Kennedy, C. J. Howard, Y. Kubota, and K. Kato, "Phase transition behaviour in the A-site deficient perovskite oxide $\mathrm{La}_{1 / 3} \mathrm{NbO}_{3}$," Journal of Solid State Chemistry, vol. 177, no. 12, pp. 4552-4556, 2004

[32] C. J. Howard, G. R. Lumpkin, R. I. Smith, and Z. Zhang, "Crystal structures and phase transition in the system $\mathrm{SrTiO}_{3}-\mathrm{La}_{2 /}$ ${ }_{3} \mathrm{TiO}_{3}$," Journal of Solid State Chemistry, vol. 177, no. 8, pp. 2726-2732, 2004.

[33] M. Kunz and I. D. Brown, "Out-of-center distortions around octahedrally coordinated $\mathrm{d}^{0}$ transition metals," Journal of Solid State Chemistry, vol. 115, no. 2, pp. 395-406, 1995.

[34] G. King and P. M. Woodward, "Cation ordering in perovskites," Journal of Materials Chemistry, vol. 20, no. 28, p. 5785, 2010.

[35] H. Moriwake, C. A. J. Fisher, A. Kuwabara, and D. Fu, "Firstprinciples study of point defect formation in $\mathrm{AgNbO}_{3}$," Japanese Journal of Applied Physics, vol. 52, no. 9S1, article 09KF08, 2013.

[36] A. Kania and J. Kwapulinski, " $\mathrm{Ag}_{1-\mathrm{x}} \mathrm{Na}_{\mathrm{x}} \mathrm{NbO}_{3}$ (ANN) solid solutions: from disordered antiferroelectric $\mathrm{AgNbO}_{3}$ to normal antiferroelectric $\mathrm{NaNbO}_{3}$," Journal of Physics: Condensed Matter, vol. 11, pp. 8933-8946, 1999.

[37] S. Miga, A. Kania, and J. Dec, "Freezing of the $\mathrm{Nb}^{5+}$ ion dynamics in $\mathrm{AgNbO}_{3}$ studied by linear and nonlinear dielectric response," Journal of Physics: Condensed Matter, vol. 23, article 155901, 2011.

[38] A. Kania, A. Niewiadomski, S. Miga et al., "Silver deficiency and excess effects on quality, dielectric properties and phase transitions of $\mathrm{AgNbO}_{3}$ ceramics," Journal of the European Ceramic Society, vol. 34, no. 7, pp. 1761-1770, 2014.

[39] J. Gao, L. Zhao, Q. Liu, X. Wang, S. Zhang, and J.-F. Li, “Antiferroelectric-ferroelectric phase transition in lead-free $\mathrm{AgNbO}_{3}$ ceramics for energy storage applications," Journal of the American Ceramic Society, vol. 101, no. 12, pp. 54435450, 2018.

[40] C. L. Farrow, P. Juhas, J. W. Liu et al., "PDFfit2 and PDFgui: computer programs for studying nanostructure in crystals," Journal of Physics. Condensed Matter, vol. 19, no. 33, p. 335219, 2007.

[41] M. G. Tucker, D. A. Keen, M. T. Dove, A. L. Goodwin, and Q. Hui, "RMCProfile: reverse Monte Carlo for polycrystalline materials," Journal of Physics: Condensed Matter, vol. 19, article 335218, 2007. 